\title{
Public involvement in health research: a case study of one NHS project over 5 years
}

\author{
Amanda Howe ${ }^{1}$, Sarah Delaney ${ }^{2}$, Jacqueline Romero ${ }^{3}$, Adele Tinsley ${ }^{2}$ and Penny Vicary ${ }^{4}$ \\ ${ }^{1}$ University of East Anglia, Norwich, UK \\ ${ }^{2}$ Graduates of the UEA MB/BS programme \\ ${ }^{3}$ NHS Norfolk Primary Care Trust, Norwich, UK \\ ${ }^{4}$ PPIRes (Patient and Public Involvement in Research) volunteer, Norwich, UK
}

Background: Public involvement, both in the National Health Service (NHS) and in clinical research, is promoted as an important democratic principle. The declared aims are to reduce professional autocracy and allow a broader ownership of the research agenda; also to improve the design of, and recruitment and retention of patients to, clinical studies. There have been a number of national initiatives in the UK to improve public input to clinical research activities, but very few reports of effective and sustainable partnerships over time. This study reports the evaluation of one example, which is embedded in the NHS and university partnerships in the Norfolk area of England. Objectives: Evaluate:

- Putting principles into practice of public involvement in research over a 5 year period for one specific project (Patient and Public Involvement in Research).

- How the model contributes to, and impacts upon, all stages of the research process.

- Attitudes of the research community and lay volunteers to their mutual experiences of public involvement.

- Key factors and strengths of this project, and areas for improvement.

Methods: A mixed methods approach related to the 5 years from start of 2003 to end of 2007. This used descriptive statistics of volunteer activity, interviews with key stakeholders (13), questionnaires (53\% response rate), and focus group with 10 volunteers to explore emergent themes. We analysed findings using a policy framework approach. Results: About 47 of the original 55 volunteers remained on the panel after 5 years. All have undertaken training, 38\% have been involved in the full range of research activities offered, and $75 \%$ have attended at least one research project meeting. Some are active in governance, ethics, and advisory committees.

Both the research community and the volunteers are very positive about the project. The researchers find it provides well prepared personnel, and gives a speedy and efficient way of fulfilling the expectations of funders for lay input. The volunteers find it gives them important opportunities to influence the quality of research and thus support improvements in patient care. Areas for improvement include increasing social diversity among the volunteers, and improving feedback on input from volunteers, without which volunteers tend to lose confidence and motivation. Conclusion: Longterm sustainable and valuable public input to research is possible. Key factors are committing resources, embedding the service in the infrastructure of a research consortium, and ongoing responsiveness by NHS staff and researchers. Additional activity to recruit and support access may be needed to attract people from a broad range of

Correspondence to: Professor Amanda Howe, Primary Care Group, School of Medicine, Health Policy and Practice, University of East Anglia, Norwich NR4 7TJ, UK. Email: Amanda.howe@uea.ac.uk

C) Cambridge University Press 2009 
sociodemographic backgrounds. Some volunteers want more involvement than this model currently offers.

Key words: improving quality; public involvement; research

Received 9 December 2008; accepted 1 September 2009; first published online 10 December 2009

\section{Background and context}

UK health service policies claim that patients and the public should be treated as equal partners in care, suggesting that historical dominance by professionals is no longer acceptable. In the context of health research, a lay perspective is expected to improve recruitment and retention of patients in research studies. As people become less likely to accept a paternalistic direction to their choices, informed consent must be coupled with an understanding of, and commitment to, research - otherwise the public might refuse consent to any research at all. Many clinical studies involve additional time and effort by patients, and, sometimes, additional risks - so an effort to improve public understanding of research underlies the interests of researchers and the National Health Service (NHS), which duly benefits from new knowledge in clinical practice.

There is emerging evidence on the benefits of public involvement. For example (Farrell, 2004), evidence collected across a number of projects found that public involvement can influence the policies, plans, and services of NHS organisations; and increase the confidence, understanding and skills of the people who participate. Benefits also included increased patient satisfaction, reduction in anxiety, greater understanding of personal needs, improved trust, better relationships with professionals, and positive effects on health. People who use services can help to ensure that research does not just measure outcomes that are identified and considered important by professionals. They can help with the recruitment of their peers for research projects; can help access people from minority and marginalised communities; and can work to ensure that results of research lead to changes (Faulkner and Thomas, 2002). There are also challenges - involving people effectively, overcoming political and societal barriers, and how to maximise positive impacts on research is still relatively under-researched, and may require cultural change on both sides (Israel et al., 1998).

While some advocate a fully reciprocal research process, where every component must be agreed with and involve the research participants (Gibson et al., 2001), the current UK government supports a strategy of 'consultation and collaboration' (INVOLVE, 2008a; 2008b). This is not as radical as user controlled research, but is a considerable improvement on a 'manipulative' model of consumer involvement, which is designed to meet the ends of those in power (Arnstein, 1969). Three main research funders of medical research (the Medical Research Council, the UK Clinical Research Collaboration, and the National Institute for Health Research) all have websites advocating and justifying active public involvement in research. Some funders will no longer accept research proposals without evidence of 'lay' input ${ }^{1}$. However, while at policy level the case is well made and the exhortations loud, there are few examples in the recent literature of operational models and their impacts in the NHS. We also know that public uptake of democratic opportunities may be less enthusiastic than expected, and may be skewed towards those with socioeconomic and educational advantage (Fotaki et al., 2005). So there is a need in the literature both for evaluations of how policy is being implemented in practice, and for critical review of how accessible these options are to the public in all its diverse forms. Evaluations should also reflect the criteria of good practice in public involvement (most clearly set out on the INVOLVE website 2008), where genuine engagement by researchers is expressed through giving time, respect, access, support, recognition and reward;

\footnotetext{
${ }^{1}$ National Institute for Health Research defines such input as from 'a patient, carer, member of an organisation or group promoting users' interests, possibly a long-term user of services or a member of the public who may be a potential recipient of a health programme' http://www.sdo.nihr.ac.uk/ ppihs1.html, downloaded 16/10/08
} 
Table 1 PPIRes - a vignette of input into one research study

\begin{abstract}
Step 1: three researchers (G.P., sociologist, and pharmacist) want to submit a 'Research for Patient Benefit' bid about adherence to medication for prevention of osteoporosis

Step 2: they email an outline of the project to the PPIRes co-ordinator, requesting volunteer input to read the draft bid and to be cited as having commented

Step 3: the co-ordinator identifies two volunteers who have expressed an interest in osteoporosis, and who are already active on the trial group of a project funded by the Medical Research Council which is looking at osteoporosis screening

Step 4: the volunteers agree to help. Their names and contact details are passed to the lead researcher, with a request from the PPIRes co-ordinator that she gets feedback about outcome of contact, and that any patient and public involvement for the bid is costed in (using rates that equate to other projects for expenses etc.)

Step 5: the volunteers' input alters the lay summary (less jargon), the aims of the interviews (putting more emphasis on practical issues such as prescription collection), and raise the issue of the 'medicalisation of old age' - this also is built into the aim as questions are broadened to address this important concept

Step 6: the research team include costs of PPIRes volunteers to be on the Study Steering Group, and now can confidently answer 'what lay input have you had?'

Step 7: wait 5 months, some revisions asked for, bid then approved

Step 8: PPIRes is informed, and the volunteers take up their ongoing role on the team.
\end{abstract}

PPIRes = patient and public involvement in research.

and by being responsive to suggestions made by lay participants. The expected gains for research include a more relevant research agenda that reflects patient experiences (Tallon et al., 2000): increased patient advocacy for research (Goodacre, 1999): and improved health literacy around the constraints of research (Ross et al., 2004).

\section{Background to the project}

The 'Patient and Public Involvement in Research' (PPIRes) project has existed since 2003, and has had a previous published evaluation in its start-up phase (Howe et al., 2006). It is supported by funding through the NHS Research and Development (R\&D) costs sustained by the Norfolk and Waveney R\&D consortium; is administrated through the R\&D office hosted by Norfolk Primary Care Trust; and has a project facilitator (at time of evaluation this was a 0.6 fulltime equivalent post) who co-ordinates all aspects of work with the public that relates to the delivery of research. The facilitator's role includes:

- Information, recruitment, selection, and training of volunteers.

- Regular support meetings - regular steering group meetings, organised visits (eg, to the Clinical Trials Unit), and specific training activities - these cover what research is, different types of study, governance and ethics: the role of lay volunteers, dealing with meetings, assertiveness, and topics of the volunteers' choosing, also discussions of experiences and shared learning.

- Support for identifying volunteers for involvement with meetings and bids when researchers request help.

- Cascading information on, and discussing research strategies and developments.

- Acting as a resource for NHS and universitybased researchers wishing to engage with the public, including giving guidance on expectations and direct seeking of volunteers.

- Acting as a link between the lay and professional experts in ongoing projects and committees.

- With the administrator, collecting information about what volunteers are doing, and collating the activity database.

- Support and contribute to ongoing evaluation of the overall PPIRes project.

One example of how PPIRes works in a research study is included (see Table 1).

The service was set up in accordance with principles of good practice in public involvement (Table 2). This work reviews activities from 2003-2007, with specific focus in some data on the 3 year period 2005-2007. The project design was developed between the PPIRes facilitator (J.R.), a PPIRes volunteer (P.V.), an academic lead from University of East Anglia (UEA) (A.H.), and two UEA medical students (S.D. and A.T.) undertaking different components as research projects.

Primary Health Care Research \& Development 2010; 11: 17-28 
Table 2 Good practice in public involvement (as implemented in PPIRes)

\begin{tabular}{|c|c|c|c|}
\hline Principle & Reference & Operationalization & Limitations \\
\hline Reimbursement & $\begin{array}{l}\text { 'Reward and Recognition', } \\
\text { Department of Health } \\
\text { CSIP (271815), } 2006\end{array}$ & $\begin{array}{l}\text { Funding available in cash for } \\
\text { travel, carer expenses, and } \\
\text { committees }\end{array}$ & $\begin{array}{l}\text { Costing of time is not equivalent to } \\
\text { employed rates: impact on } \\
\text { benefits can be a problem }\end{array}$ \\
\hline $\begin{array}{l}\text { Principles of } \\
\text { effective user } \\
\text { management }\end{array}$ & $\begin{array}{l}\text { SDO briefing paper 2004, } \\
\text { 'How Managers Can Help } \\
\text { Users to Bring About } \\
\text { Change in the NHS' }\end{array}$ & $\begin{array}{l}\text { Clear role description; } \\
\text { guidance notes for } \\
\text { volunteers and staff, and } \\
\text { Training and support }\end{array}$ & $\begin{array}{l}\text { Relies on all involved to use these } \\
\text { and to comply with them }\end{array}$ \\
\hline $\begin{array}{l}\text { Effective } \\
\text { implementation } \\
\text { of public } \\
\text { involvement }\end{array}$ & $\begin{array}{l}\text { 'Diffusion of Innovations', } \\
\text { SDO Literature Review }\end{array}$ & $\begin{array}{l}\text { Attempt to match the project } \\
\text { to the needs of NHS and } \\
\text { research }\end{array}$ & $\begin{array}{l}\text { Changing expectations; tensions } \\
\text { because of control by researchers }\end{array}$ \\
\hline Ethical practice & Entwistle et al. (1998) & $\begin{array}{l}\text { Training for volunteers to } \\
\text { become alert to ethical issues }\end{array}$ & $\begin{array}{l}\text { Presence of lay people does not } \\
\text { guarantee ethical practice }\end{array}$ \\
\hline $\begin{array}{l}\text { Core expectation } \\
\text { for research } \\
\text { process }\end{array}$ & $\begin{array}{l}\text { Best Research for Best } \\
\text { Health; Department of } \\
\text { Health (272605), } 2006\end{array}$ & $\begin{array}{l}\text { Guidance notes for; } \\
\text { volunteers, and staff } \\
\text { training and support }\end{array}$ & $\begin{array}{l}\text { Developing applied understanding } \\
\text { takes time and experience }\end{array}$ \\
\hline
\end{tabular}

PPIRes = patient and public involvement in research; NHS = National Health Service; SDO = service delivery and organisation.

The students were supervised by Amanda Howe, and their work received ethical approval by the UEA Faculty of Health Ethics committee in November 2007.

\section{Methods}

The aims of the project were to evaluate the efforts to 'put principles into practice' in public involvement research over a 5 year period in one specific project (PPIRes) by

- Describing who was currently active on the volunteer panel (including their sociodemographic profile), and review reasons for dropping out.

- Summarising the activities of the volunteers over the last three complete years.

- Analysing the strengths and weaknesses of PPIRes as perceived by the R\&D community (academics and managers), and the PPIRes volunteers.

- Developing a critical summary of impacts to date and next steps.

The stages of data collection of the project were as follows:

1) Review of existing database by PPIRes coordinator and R\&D staff, to summarise characteristics and activity of PPIRes volunteers.

2) Summary of relevant policy and literature.
3) Initial interviews with key academics and NHS managers involved with PPIRes.

4) Evaluation questionnaire to all volunteer panel members.

5) Focus group of volunteers to explore issues arising from stages $1-4$, and validate recommendations for future action. ${ }^{2}$

Framework analysis (Ritchie and Spencer, 1994) was used as an analytic approach, to allow specific themes set out in the aims of the project to be pursued across different datasets and researchers. The overall theoretical approach of the project was action research oriented, using each stage to raise awareness and feed ideas back into the overall evaluation, with a final iterative cycle to draw out potential actions. This has been put into a formal report for local consortium members and volunteers, as well as being opened to external review through this study.

\footnotetext{
${ }^{2}$ Amanda Howe, Jacqueline Romero, and Penny Vicary developed ideas for the project and details of the themes to be explored at all stages: Jacqueline Romero provided personnel and activity data for the volunteers: Sarah Delaney undertook and analysed the interviews with researchers and managers: Adele Tinsley developed the questionnaire with Jacqueline Romero and Penny Vicary, and collected and analysed these data: Amanda Howe led the focus group with Jacqueline Romero and Adele Tinsley, which Amanda Howe and Adele Tinsley analysed: Amanda Howe led the design and writing up of the project.
} 


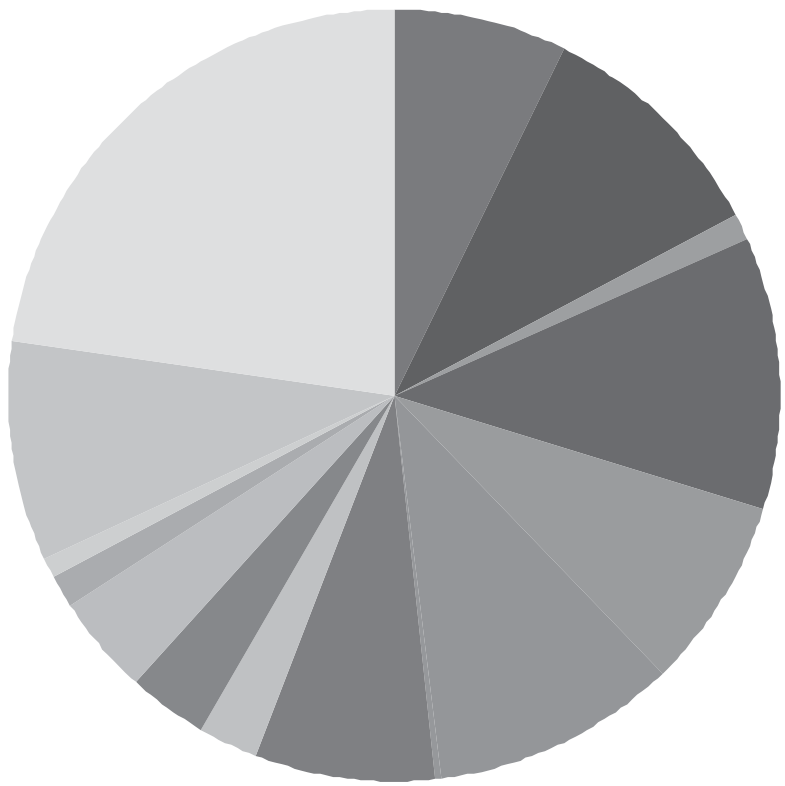

\author{
Help with funding application \\ - Reviewing submitted bids \\ Guidance on consent \\ Advice on Information Sheet \\ Steering Group \\ - Advice on Lay Summary \\ - Dissemination findings \\ - Committee Membership \\ - Other advice / input \\ Questionnaire Design \\ Attendance at conference \\ - Speaker at meeting \\ Leaflet design \\ - Pre-bid discussion / advice \\ General Enquiries
}

Figure 1 Range of activities and proportion of volunteers involved over 3 years

\section{Results}

\section{The volunteers}

From the original 55 volunteers recruited in 2003-2004, 47 remain on the database, with two choosing to be currently classified as 'nonactive'. The eight who have left gave 'moved away' or personal commitments as the reason. About 42 of the volunteers are female, five are male: the majority are over 60 years of age and declare their ethnic background as White British. The range of research projects covers the whole of the consortium and university health portfolio where patient or staff recruitment is involved, the majority being projects with a direct patient and clinical emphasis. Involvement in epidemiological and laboratory-based studies is much less common.

A summary of the volunteer activities shows that:

- All volunteers have attended one or more PPIRes training and support meetings.

- $38 \%$ have undertaken a full range of possible activities (see Figure 1).

- $96 \%$ have commented on participant information sheets, $88 \%$ commented on lay summaries, and $75 \%$ have attended at least one research project meeting or steering committee.

- All requests for involvement have received a response by a volunteer within acceptable timeframes.

- Some of the volunteers have been active at regional and national levels, for example presenting at conferences or working with the national Clinical Research Networks.

- However, less than $20 \%$ have been named on grants in the period under study.

Figure 1 is based on 218 episodes of activity over a 3 year period, and shows the range of activities undertaken, including ongoing committees and steering groups.

\section{The interviews}

Interviews were conducted with professionals involved with health research. This was a purposive sample, which included NHS R\&D managers, academics who had helped to create the PPIRes project (and might therefore be seen as 'advocates'), plus local researchers who had been users/recipients of PPIRes volunteers on one or more bid. Thirteen potential participants were approached to take part in the study, all of whom 
agreed. Signed informed consent to a taped interview was obtained from each participant. Semistructured interviews were carried out, which were recorded and transcribed verbatim. Transcripts were collated and thematically analysed. Eleven of the participants chose to review transcripts of their interviews, and to receive the results.

Of the 13 interviewees, 8 were female. Seven held fulltime university positions, of whom three were clinical academics. Four had been involved with the start of PPIRes. Two currently held NHS R\&D managerial and support posts, while all others had worked with PPIRes volunteers in a research capacity. While managerial perspectives tended to highlight policy issues, and researchers were more focussed on the needs for public involvement as a means to funding and recruitment, the majority of respondents were united in highlighting the value of efficiency and the speed of turnaround of queries (eight participants), usually in the context of research being a time-pressured activity:

...As a researcher you know, to have to even think about finding people, sorting out whether they're the right people, talking to them about how research works, giving them some money to come to a meeting, you know I don't need it. Life's too short. This is like an expert group who does that. And does it very well in my experience.

All researchers mentioned using PPIRes volunteers within a variety of settings for their projects: one example included the role of two volunteers on a successful bid for a project doing a randomised controlled trial of asthma 'at risk' registers where they helped to develop the bid, assisted with the information sheets for participants, and were now part of the Trial Management Group:

They can bring things to a project, things that you didn't necessarily think of.... how it's likely to impact on how easy it is to do the research, or how do you get the quality of the information that you get from patients....I think researchers are sometimes quite blindfold to the fact that there are these sorts of practical issues that they need to take into account.

They commended the volunteers for their valuable role in developing lay summaries, reviewing patient information sheets, and contributions to steering groups. A project on nutrition in the elderly had had volunteers who were:

involved from the very conception of it. They really worked it up before it went to funding, and in fact I think we almost certainly wouldn't have got funding if they hadn't of been involved. ... ... they've had an overview role of the whole study so we've had meetings every couple of months since we started the grant to make sure they know what's happening all the time, also for the other members of the steering group too. But also they'be been very involved in very helpful things. Like when we found recruitment quite difficult they had lots of avenues to help us find other people to participate in the study.

Three participants also explored the role of lay volunteers in formulating questions for a research agenda. However they recognised there were practical difficulties, both because researchers usually have a research question before recruiting the lay representatives to the study, and because questions generated may not fit the requirements of funding bodies, which tend to specify the research question. One researcher also highlighted PPIRes' role in the dissemination of information, saying that volunteers were helpful in coming up with information on how to target prospective study participants. Additionally it was suggested that lay people knew where to disseminate study findings so that they would reach relevant sectors of the community.

One participant who helped to set up the organisation explored the benefits of PPIRes in terms of enhancing the consortium's research profile, where it was used as an example of a successful provision for patient and public involvement (PPI) in order to secure funding. It was also seen as helping to make the local consortium competitive against others, and its loss might lead to less success in getting national funding:

It could be disastrous in terms of research profile. You could start not getting your money because of it, which means you can't do your research, so it would be worse for patients ultimately.

Only three participants (two of whom were involved in research management as opposed to 
researchers) described any benefits for the lay volunteers. One spoke of their build of confidence over time through empowerment and support. However, the interviewees also expressed some concerns about barriers to maximal public involvement: some of these were theoretical, but some were based on recent experiences. The commonest concerns were that using lay volunteers slowed down the pace of work: it was difficult to make their involvement genuine: that the expertize of the volunteers might be inappropriately used (eg, attending a whole meeting when many items on the agenda did not involve them): problems with paying their expenses before funding was secured: and the possibility of difficulties matching the needs of projects with the personal profiles of available volunteers. ${ }^{3}$ Managerial staff and those involved in project setup were more enthusiastic about the benefits of the project, and some were critical of the research community, who were seen as sometimes being resistant to public involvement. However, all those researchers interviewed who had used the PPIRes volunteers declared themselves persuaded of the value of this project, and there were no substantive criticisms from any of the interviewees. This suggests that the model is fit for purpose, though some specific recommendations for quality enhancement can be summarized from these data as:

- Need to continue to educate the research community about PPI in general, and PPIRes in particular: a more active approach was needed to marketing and promoting PPIRes by those in academia and R\&D. The implication here was that this needed to be ongoing/ recurrent, in order to keep the issue high on the agenda and influence new researchers as they become active in the consortium.

- Creating a routine system of activity monitoring and feedback from researchers who have used PPIRes.

- Monitoring numbers and demographics of PPIRes participants, including why people leave.

- Greater transparency for how PPIRes profiles and selects volunteers: more tailoring of the

\footnotetext{
${ }^{3}$ There was no evidence from the database that any project had not received a response from PPIRes, so the basis for this perception may relate to specific views on 'suitability'.
}

mix of patients and public for projects, to the researchers' needs.

- Continuing recruitment, especially of people or agencies to work with projects where more sociodemographic diversity is relevant.

- Need to secure and expand current funding to allow more work.

\section{The questionnaire}

In order to allow all volunteers the option to contribute their views, a questionnaire was designed which was piloted with a few PPIRes volunteers before distribution to all current volunteers. The response rate after two reminders was $53 \%$. Given the homogenous sociodemographic background of the volunteers it is likely that these people were broadly representative of the volunteers as a whole.

All respondents had undertaken some form of activity as a PPIRes volunteer. Nine respondents had undertaken all the activity options (9/ $24=38 \%$ ). The most common activity undertaken was giving advice on patient information sheets for study participants $(n=23,96 \%)$. The least undertaken activity was attending training activities with other agencies $(n=12,50 \%)$. The other options for activities given were; training activities for volunteers run by PPIRes $(n=18$, $75 \%)$, advice on lay summaries $(n=21,88 \%)$, being a member of a specific research project committee or steering group $(n=18,75 \%)$, and attending a patient and public involvement conference $(n=19,79 \%)$. Other activities described were; ethics committee membership, research governance committee membership, article writing, and attendance at student learning events. The mean number of activities per volunteer over the period of 3 years was 3 (a relatively low overall demand per person). A total 91\% (21/23) of respondents rated their overall involvement and the activities undertaken as satisfactory or very satisfactory. No respondent reported this as unsatisfactory.

The most common reason for being in PPIRes was an interest in research, with a particular desire to improve research to benefit patients, and to use their own experiences of services and research participation and skills in a valuable way. Personal learning was less important, but new opportunities to meet people 
and develop a new interest were also seen as important:

Adds a further dimension to the approach used by medics - past experience suggests that the patients/carers view not considered and felt this could improve outcomes.

Wanted to improve approach and attitudes of health researchers re involving members of the public.

I would like to improve my general knowledge of the NHS and possibly contribute as a service "user".

The majority of respondents $(79 \%)$ were very happy with the support they had received from the staff of the PPIRes project, and also (89\%) with the attitudes of researchers with whom they had interacted. Their main concern was that they received insufficient feedback for many of their inputs, and this was subsequently echoed in the focus group. Examples of views were:

- 'No feedback on the value of my contribution, am I being helpful or not?'

- 'We hope to have feedback on the research we have been involved with'

- (Need)'feedback when involved in projects or general comments about response to information sheets and lay summaries.'

- 'I would like more information and updates on projects and progress, and feedback on the value of the contribution volunteers make when commenting on protocols. 4

PPIRes aims to provide out of pocket expenses for volunteers in cash and at the time of the meeting, for example travel costs and costs for the provision of a carer when necessary. A total $96 \%$ $(23 / 24)$ of the respondents felt they received adequate financial support during their involvement in PPIRes '(it) is much appreciated and makes my contribution feel valued'

However, one respondent ticked both yes and no, with one comment that the expenses were 'basic and barely covered travel'..., and others expressed some variations and concerns:

I have always received adequate financial support except for (one) committee which

\footnotetext{
${ }^{4}$ Since the data was collected the feedback process has been revised.
}

Primary Health Care Research \& Development 2010; 11: 17-28

\section{.... I felt it might put some members off being involved}

(we) need to make researchers aware of the financial and time costs of the patient and public involvement if collaboration is to be not just a token gesture."

There were two recurrent themes about how the project could be further improved: one focused on a desire for more involvement with the research community (problems of low uptake and lack of feedback) and the other on a desire to increase the profile of the project, its funding and staffing, and recognition of its potential benefits for individuals, research, and the NHS.

\section{Focus group}

A focus group of 10 volunteers ( 9 female, 1 male, all were White British) was held to consider some of the issues raised in the previous data collections. These individuals were invited on the basis of their expressed consent to attend at the time of returning the questionnaire. Prompts provided by the chair included the issues of the restricted sociodemographics of the group, the perceived lack of feedback from researchers, and the effectiveness of current and future means to maximise volunteer support.

All volunteers spoke during the session: a few direct questions were asked of the PPIRes facilitator and the chair. The interpersonal dynamics suggested a group who knew each other relatively well, and who had shared assumptions about some of the discussion. The points summarized below had a high level of consensus.

1) Making it possible for lay people to 'have a voice at the table'. PPIRes was seen as having a crucial role in providing this, both by getting access but also assisting effective contribution. The training and support functions of the project were seen as essential to realising public involvement, and giving the volunteers increased confidence to question and make suggestions. This growing confidence was built up over time, with increased activity, and with positive feedback.

2) The ethos - research was seen as a shared endeavour, where the public had a right to be involved, and where mutual respect was seen as a precondition of success. 
3) The importance of having continuity of lay input throughout the research process - being proactive rather than reactive, getting a full understanding in order to be effective, and developing relationships with staff and other participants. Another valued aspect of continuity was the interpersonal contact - the PPIRes 'one-stop shop' with its consistency of service and contacts, plus relationships made among volunteers.

4) Process issues and good practice - volunteers were very alert to the need repeatedly to simplify language and concepts - to remind researchers that lay people would need this, and to avoid jargon and acronyms. Some key principles of inclusion were reiterated being introduced in meetings, having name tags/lists of attendees, being briefed, being able to be open about uncertainty, and giving support.

5) Facilitating diversity - the group felt that without PPIRes the public involvement in research in the region would be much less, as people who would independently feel able to contribute would be few. However, the volunteers themselves were concerned about all being white and older than the social average for Norfolk, and made suggestions about expanding the database by contacting charities with a view to 'affiliation' (willingness to be approached for relevant research, with a commitment from PPIRes to support anyone they put forward); offering 'research buddies'; and doing specific outreach to communities not represented in the current panel.

6) Problems - The main negative statement was about lacking feedback from researchers - it was strongly felt that every contact/episode of activity should result in some feedback for the recipient. Without such audit and monitoring, the volunteers felt it was impossible to judge the value of their personal and collective efforts. Other concerns were about whether the funding and opportunities for PPIRes would be sustained - or, they hoped, increased. Some volunteers felt underused, and felt they could contribute more if asked more often to assist; they also were more motivated to contribute if they could see, and learn from, the impacts of their role.
Suggestions to the R\&D office for more effective working of the project are therefore summarized as:

- More information to researchers on the existence and use of the PPIRes volunteers, with some minimum requirements for uptake where appropriate

- Better monitoring of activity episodes and their value and outcomes, with feedback to volunteers and researchers: this needs to made routine, so that all contacts between researchers and volunteers is recorded and evaluated

- More outreach to agencies and communities currently under-represented in PPIRes, with tailored trainings and support to improve their input as needed.

The implications of these suggestions support the potential need for increased staff resources, and might address the desire for increased scope of volunteer activity.

\section{Discussion}

It is clear that the PPIRes model has had a sustainable input to the research community over the last 5 years, with considerable effort, structural and financial investment, and cultural change. It is worth noting that, from 2007 onwards additional infrastructure money was secured to extend the panel's coverage into Suffolk - which can be seen as a sign of the acceptability and importance of its activities. Nevertheless, even with this concerted effort, the processes of involving the public in research are not yet fully normalized (May et al., 2007). Three disjunctions are suggested by these data - some researchers may not be using, or are under using, the public in their research processes: even if they engage, the routine processes of this R\&D project do not fully capture and feedback the impacts of these encounters: and the full range of public voices needed to represent contemporary society do not spontaneously engage through a panel based on an individual volunteering culture, even when active support, training, and personal expenses are offered.

Further research is needed on the reasons why researchers may not take up public involvement options, and the extent to which researcher expectations of volunteer demographics are 
influenced by social stereotypes. Similarly, the perceived lack of feedback from researchers to volunteers might be due to the longer timeframe of research ('I haven't heard back from the funders yet so I didn't have any feedback to give'), but there appears to be a need to improve researcher responsiveness to the input of the public. Attempts to retain both individuals and agency links are likely to be weakened if people feel underused, so any new recruitment drive to increase range of volunteers will need matching by researcher uptake and responsiveness.

There is another discussion point, which concerns the expectations of members of the public for increasing their ability to lead the research process. PPIRes was set up to improve the quality of the overall research process rather than train volunteers to do research, and user led research may not improve the overall quality of NHS research; but for some, the option to do research themselves may be important within PPIRes for encouraging and retaining volunteers.

Extending the volunteer database both in quantity and in social background may mean more reciprocity in setting research priorities and allowing volunteers greater scope in the development of research - both potentially time consuming and politically more demanding - but the literature suggest that as broad and flexible an approach as possible will maximise user involvement, and INVOLVE point out that minority groups are: 'much more likely to work with researchers who want to collaborate with them on research that has been identified by the community as a priority'.

There are limits to this study: it is only one example, with its own local constraints and opportunities. The response rate was low in spite of several verbal and written reminders - this may be because it was seen as coming from 'outside' the group (sent from UEA), which reduces obligation but may also reduce responsiveness. It was disappointing that in spite of this extensive data collection and the evidence of considerable activity and widespread satisfaction with PPIRes, there was no really robust evidence on the impacts of the volunteer inputs on the overall quality or quantity of funded research. This again makes the case for a much stronger monitoring of PPIRes activity, and feedback on the outcomes of grants: it also shows that there is more research to be done on how to judge effective outcomes from public involvement with research. Other work also suggest that it is easier to agree on process indicators (eg, activity data, levels of input) than outcomes (eg, increased success in getting funded, Cooper et al., 2008), and a stronger study design would be needed to know whether specific components of any intervention are key to the outcomes of research over time.

The extent to which the lessons from the PPIRes model and its current development can be useful to others depends on the extent to which they have common contexts and goals. Within the UK we would argue that there are transferable lessons to be learned by others committed to the effective delivery of public involvement in research. The context has changed rapidly over the period of the study. For example, some funders now permit applications for 'open' funding - for example the research for patient benefit stream of the NHS R\&D does not specify research questions, so this has opened the scope for members of the public to help to set the research agenda. The regional components of the UK Clinical Research Network (UKCRN) are setting up PPI activities, which draw on PPIRes and may strengthen its position. National developments in PPI may also lead to embedding of the principles espoused by this project, which should help researchers to be more responsive.

\section{Conclusion}

This study suggests that it is possible to create a sustainable core service to researchers to ensure public involvement in research and development. There are four clear recommendations for enhancing public involvement in any such project:

- Ensure recurrent and targeted marketing of any PPI R\&D model to both the research community and public, to ensure full usage and new involvement - volunteers are well placed to promote their own work and to champion it.

- Extend volunteer recruitment by securing and retaining links with other community agencies and groups with diverse populations.

- More systematic evaluation - embed strong routine follow-up for any and all activities and 
contact episodes, getting and giving feedback to both researchers and volunteers. This will make the outcomes of the PPIRes input clearer, and avoid the feeling of not knowing whether input has been useful or not. ${ }^{5}$

- Include funding in infrastructure R\&D monies for a service where staff provide a local support and development unit for PPI - it is likely to prove both effective and efficient.

This project shows that with systematic planning, consistent but modest pump priming funds, and a political commitment, an R\&D consortium can create and retain a robust model of patient and public involvement in research. The principles of good practice in involving individuals are already described. $^{2}$ This study shows how a local R\&D system can support such good practice, and highlights some further systems factors that may lead to a comprehensive service, which can satisfy the needs of most researchers and members of the public. Early commitment to detailed audit of contacts and outcomes, and to the need to search out volunteers from minority backgrounds via appropriate agencies, may avoid social biases in the available volunteers. There is enormous goodwill and expertize in the public towards health research, and researchers can learn to use this appropriately when supported by dedicated staff who have the trust of both the public and academics, and who have the time and commitment to facilitate both groups. A flexible range of options and a moderate but consistent flow of opportunities will be enough for most volunteers, with more substantive options (committees, user led research) being important to the motivation and commitment of a few. This kind of commitment should be possible throughout the UK given the large amount of R\&D funding now distributed through the UKCRN, and we hope to see more such models described and compared over time.

\section{Acknowledgements}

The authors gratefully acknowledge the work done for PPIRes by the volunteers, including their contribution to questionnaires and focus group

\footnotetext{
${ }^{5}$ Since the data was collected the feedback process has been revised.
}

for this study: the Norfolk PCT R\&D office (Tracy Shalom, Barbara Staffa, and Clare Symms); the longstanding commitment of Helen MacDonald (East of England Primary Care Research Network): the funding and support of the Norfolk and Waveney R\&D consortium, especially Richard Reading \& David Scott; and the support of UEA colleagues, especially Fiona Poland and Ian Harvey. We are also grateful to those who were interviewed, and those who commented on drafts of this study, including Ailsa Harpur, Chair of the RCGP Patient Partnership Group.

\section{Conflict of Interest}

Mrs Jacqueline Romero is employed as the PPIRes co-ordinator by Norfolk PCT on behalf of the R\&D Consortium.

\section{References}

Arnstein, S. 1969: A ladder of participation. Journal Royal Town Planning Institute 57, 176-82.

Cooper, C., Barber, R., Parry, G. and Boote, J. 2008: Report of an Expert Workshop on the Impacts of Public Involvement in Health and Social Care Research. ScHaRR, University of Sheffield. Retrieved 15 October 2008 from http:// www.shsrc.nhs.uk/Documentsforweb/ConConf/2007\%20Conf/ Presentation/C716\%20-\%20Expert\%20Workshop\%20on.ppt

Department of Health. 2006: Best research for best health: a new national health research strategy. The NHS contribution to health research in England. London, UK: Department of Health.

Entwistle, V., Renfrew, M., Yearley, S., Forrester, J. and Lamont, T. 1998: Lay perspectives: advantages for health research. British Medical Journal 316, 463-66.

Farrell, C. 2004: Patient and public involvement in health: the evidence for policy implementation. A summary of the results of the health in partnership research programme London, UK: Department of Health.

Faulkner, A. and Thomas, P. 2002: User-led research and evidence based medicine. British Journal of Psychiatry 180, $1-3$.

Fotaki, M., Boyd, A., Smith, L., McDonald, R., Roland, R., Sheaff, R., Edwards, A. and Elwyn, G. 2005: Patient choice and the organisation and delivery of health services: scoping review. Report for the National Co-ordinating Centre for NHS Service Delivery and Organisation R\&D. London, UK: NCCSDO.

Gibson, N., Gibson, G. and Macaulay, A.C. 2001: Communitybased research: negotiating agendas and evaluating outcomes. In Morse, J., Swanson, J., and Kuzel, A.J., editors. The nature of qualitative evidence. California, USA: Sage Publications, 160-82. 
Goodacre, H. 1999: Involving patients in clinical research. British Medical Journal 319, 724-25.

Howe, A., Barrett, B., Little, B. and MacDonald, H. 2006: Ensuring public and patient participation in research: a case study in infrastructure development in one UK research and development consortium. Primary Health Care Research and Development 7, 60-67.

INVOLVE 2008a. Retrieved 9 September 2008 from http:// www.invo.org.uk/

INVOLVE 2008b. Retrieved 15 September 2008 from http:// www.invo.org.uk/pdfs/GoodPracticeD3.pdf

Israel, B.A., Schulz, A.J., Parker, E.A. and Becker, A.B. 1998: Review of community-based research: assessing partnership approaches to improve public health. Annual Review of Public Health 19, 173-202.
May, C., Finch, T., Mair, F., Ballini, L., Dowrick, C., Eccles, M. et al. 2007: Understanding the implementation of complex interventions in health care: the normalization process model. BMC Health Services Research 7, 148. (2007 doi:10.1186/1472-6963-7-148).

Ritchie, J. and Spencer, L. 1994: Qualitative data analysis for applied policy research. In Bryman, A. and Burgess, R.G., editors. Analyzing Qualitative Data. London, UK: Routledge, 173-94.

Ross, F., Donovan, S., Brearley, S., Victor, C., Cottee, M., Crowther, P. and Clark, E. 2004: Involving older people in research: methodological issues. Health and Social Care in the Community 13, 268-75.

Tallon, D., Chard, J. and Dieppe, P. 2000: Consumer involvement in research is essential. British Medical Journal 320, 380. 